\title{
Translocation of particles deposited in the respiratory system: a systematic review and statistical analysis
}

\author{
Hideo Nakane
}

Received: 1 August 2011/Accepted: 28 October 2011/Published online: 20 November 2011

(C) The Japanese Society for Hygiene 2011

\begin{abstract}
Many epidemiological studies have demonstrated that ambient particulate matter poses consistent risks for respiratory and cardiovascular disorders. The translocation of inhaled particles is one hypothesis that could explain such systemic effects. The objectives of this study were to conduct a systematic review of previous reports on particle translocation from the respiratory system and to discuss factors important for translocation. A PubMed search was conducted in August 2011 for the period from 1967 with four main keyword domains (particle, translocation, detection site, and exposure route). The systematic review identified 61 original articles written in English that met the specified criteria (i.e., information on experiment and particle detection). Categorical regression analysis was performed with the site of particle detection as the objective variable, and particle size, particle material, animal species, and exposure route as the explanatory variables. All explanatory variables showed statistically significant effects. The effects for particle size and particle material were large, while the effects for animal species and exposure route were relatively small. There was a broad relationship between particle size and detection site: $\leq 50 \mathrm{~nm}$ for brain and remote organs; $\leq 1 \mu \mathrm{m}$ for blood; and $\leq 10 \mu \mathrm{m}$ for lung tissues. However, these results should be considered within the context of several limitations, such as deficiency of information.
\end{abstract}

Keywords Particle - Translocation - Respiratory system . Systematic review $\cdot$ Categorical regression

H. Nakane $(\square)$

Atmospheric Environment Laboratory,

Toyota Central R\&D Labs., Inc, 41-1 Yokomichi,

Nagakute, Aichi 480-1192, Japan

e-mail: e1050@mosk.tytlabs.co.jp

\section{Introduction}

A number of epidemiological studies have shown that airborne particulate matter (PM) is associated not only with respiratory diseases but also with cardiovascular diseases [1-3]. The existence of very small particles with diameters of $<100 \mathrm{~nm}$ (i.e., ultrafine particles; UFPs) could explain, at least in part, the effects of airborne PM on the cardiovascular system $[4,5]$. Predictive mathematical models for the deposition of inhaled particles indicate that particles of $<100 \mathrm{~nm}$ are commonly deposited in the alveoli $[6,7]$. The high surface-to-volume ratio of UFPs allows them to adsorb large amounts of substances, including various organic and inorganic compounds that induce oxidative stress and/or inflammation [8]. Inhalation of UFPs therefore triggers inflammatory responses in the lung and increases the release of inflammatory mediators into the blood. This, in turn, can lead to various changes in the cardiovascular system, such as an increase in blood coagulability and the progression of atherosclerotic lesions $[1,4]$.

In addition to such indirect effects via inflammatory responses, UFPs may directly affect the cardiovascular system by translocation from the respiratory system to the systemic circulation [9]. UFPs deposited in the lung may pass through the epithelial barrier because of their very small size; some particles may move into lung capillaries and then into the systemic circulation. Furthermore, inhaled UFPs could migrate to the brain from the nasal cavity via the olfactory nerve [9].

To evaluate the possibility of particle translocation from the airway or alveolar lumen, various studies have been conducted in which artificial particles were inhaled or administered into the airway. Afterwards, various organs or tissues, including the lymph nodes, blood, liver, heart, spleen, kidney, and brain, were examined for the presence of 
particles. Although several review articles concerning particle translocation have been published [9-12], no systematic reviews have been performed. In addition, important factors that affect inhaled particle translocation could be identified by a multivariate analysis of integrated data on particle translocation. Therefore, the purposes of this study were to summarize the studies published to date on particle translocation from the respiratory system, and to discuss factors important for the translocation of solid particles. In the literature search for the present study we did not limit particle size because it was felt that information on the translocation of large particles (i.e., $>100 \mathrm{~nm}$ ) would be useful for the subsequent statistical analysis. To the best of the authors' knowledge, this is the first systematic review of particle translocation including statistical examination.

\section{Materials and methods}

Data source and keywords for literature search

A systematic search of the PubMed database (http:// www.ncbi.nlm.nih.gov/sites/entrez) was performed for articles published in peer-reviewed journals from February 1967 through August 2011. The search was conducted using four main keyword categories, with terms related to: (1) particle (i.e., particle, particulate, nanoparticle, or *sphere); (2) translocation (i.e., pass, passage, translocat*, move*, migrat*, transport*, or incorporat*); (3) detection site (i.e., systemic, circulat*, blood, brain, liver, lymph*, nerv*, epithel*, or interstiti*); and (4) methods by which particles were exposed or the site into which particles were administered (i.e., inhal*, intratracheal*, respir*, airway, trache*, bronc*, alveol*, lung, nose, nostril, or nasal).

\section{Selection of studies}

Five main criteria were used for study selection.

1. Type of report: Original reports written in English.

2. Design of experiment: Animal or human studies in which particles were either inhaled or administered into the airway. Neither in vitro studies nor studies using isolated respiratory organs were included.

3. Information on experiment: Material and size of the particle, animal species, route of exposure, and the major results were specified. Major results should be shown with figures, tables, or photographs. Information on the particle concentration and duration in inhalation experiments or the amount of administered particles in administration experiments was not required, but was included for reference.
4. Detection of solid particles: Studies focusing on the translocation of solid particles. In reality, the constituents of the particles may dissolve or labels may be released from the particles. Such soluble fractions pass the epithelial barrier more easily than solid particles. An attempt was therefore made to exclude the cases where soluble fractions were detected. More specifically, papers were excluded in which such possibilities were pointed out by the authors themselves or by other researchers.

5. Validity of detection: Reports in which translocated particles were detected were evaluated for the validity of detection. Data that met at least one of the following four additional criteria were regarded as having successful detection at the translocated sites: [A] particles were observed histologically in the target tissue; [B] statistically significant amounts were detected in the target tissue; $[\mathrm{C}]$ a dose-response relationship was observed for more than two different doses or concentrations; or $[\mathrm{D}]$ more than $0.1 \%$ of the administered dose or lung deposition was observed in the target tissue.

\section{Statistical analysis}

Categorical regression (CATREG) analysis was performed using the studies that met the criteria described in the previous section. The objective variable was the site where particles were detected, and the explanatory variables were particle diameter, particle material, animal species, and exposure route. Doses or concentrations were not adopted as the explanatory variables for the following reasons: (1) there were several reports in which such information was not available; (2) varying descriptions were used in the inhalation studies (e.g., initial lung burden or initial body burden instead of concentration and time); and (3) subgroup analyses would have been required because of the difficulty in standardizing the exposure conditions in different styles of experiments, but there were not enough data to conduct such analyses.

The sites where particles were detected were classified into five nominal categories: (1) no translocation (remained in the lumen of the airways or alveoli); (2) lung tissues (in the epithelial cells of the airways or alveoli, submucosal tissue, interstitium, lung-associated lymph nodes, or endothelial cells of capillaries); (3) blood (in pulmonary capillary lumens, systemic blood circulation, liver, or spleen); (4) remote organs (organs other than lung, liver, spleen, or brain); and (5) brain (olfactory bulb, cerebrum, limbic system, brainstem, or cerebellum). The liver and the spleen were categorized as "blood" because these organs inherently function as eliminators of particles from the blood. Studies in which particles could not be detected in certain tissues were not included in the statistical analysis 
and were considered "negative results." However, in studies in which particles were not detected in the lung tissue, the results were adopted as "no translocation." Studies in which particles were detected in alveolar macrophages were also classified as "no translocation" because alveolar macrophages phagocytize foreign materials in the alveoli, and most of the cells migrate to terminal bronchioles, where they are cleared by the mucociliary escalator.

Particle diameter was classified into five ordinal categories: (1) $\leq 50 \mathrm{~nm}$ (nanoparticles); (2) $>50$ and $\leq 100 \mathrm{~nm}$ (UFPs without nanoparticles); (3) $>100$ and $\leq 1 \mu \mathrm{m}$ (accumulation mode particles); (4) $>1$ and $\leq 10 \mu \mathrm{m}$ (inhalable particles); and (5) $>10 \mu \mathrm{m}$ (non-inhalable particles). Particle material was classified into six nominal categories: (1) heavy metals and salts [gold $(\mathrm{Au})$, silver $(\mathrm{Ag})$, platinum $(\mathrm{Pt})$, copper $(\mathrm{Cu})$, iridium $(\mathrm{Ir})$, tantalum (Ta), and barium sulfate $\left.\left(\mathrm{BaSO}_{4}\right)\right]$; (2) metal oxides [manganese dioxide $\left(\mathrm{MnO}_{2}\right)$, trimanganese tetraoxide $\left(\mathrm{Mn}_{3} \mathrm{O}_{4}\right)$, cadmium oxide $(\mathrm{CdO})$, titanium dioxide $\left(\mathrm{TiO}_{2}\right)$, iron(II,III) oxide $\left(\mathrm{Fe}_{3} \mathrm{O}_{4}\right)$, and cerium(IV) oxide $\left.\left(\mathrm{CeO}_{2}\right)\right]$; (3) inorganic carbons (carbon black, C60 fullerene, and carbon particles generated with a Palas ${ }^{\circledR}$ (Palas, Karlsruhe, Germany) aerosol generator); (4) silicates (fly ash, silica, and aluminosilicate); (5) plastics [polystyrene and its conjugates, Teflon ${ }^{\circledR}$ (DuPont, Wilmington, DE, USA), polyacrylamide, polylactic acid, and methoxypoly (ethylene glycol) (mPEG)]; and (6) proteins (albumin and ferritin). $\mathrm{BaSO}_{4}$ had only one datum; it was combined with heavy metals because a preliminary CATREG analysis indicated that quantification of $\mathrm{BaSO}_{4}$ was similar to that of heavy metals. Animal species was classified into four nominal categories: (1) rat; (2) mouse; (3) guinea pig and hamster; and (4) dog. There were no human data available for CATREG analysis because all the data showed negative results. There was one datum each for guinea pig and hamster; these data were combined because a preliminary CATREG analysis indicated that quantifications of these species were similar. Exposure route was classified into three nominal categories: inhalation, intratracheal administration, and intranasal administration.

Statistical analysis was performed with IBM SPSS Statistics v. 19 (IBM, Armonk, NY, USA) and IBM SPSS Categories v. 19 (IBM).

\section{Results and discussion}

\section{Selection of studies}

The literature search was conducted in August 2011. Eight hundred thirty-seven reports met all four main subject heading domains. Figure 1 illustrates the selection process. First, based on their titles and abstracts, 709 articles were

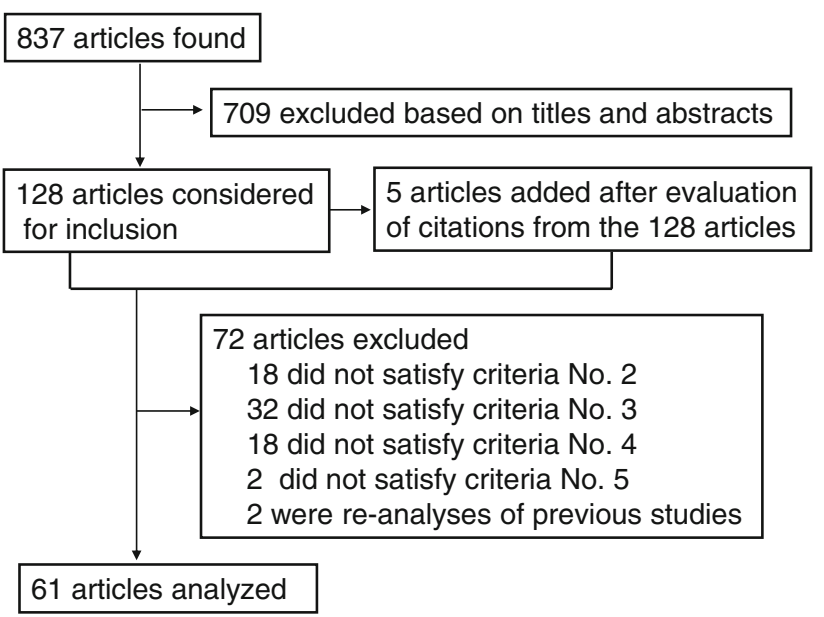

Fig. 1 Flow diagram of the study selection process. Criteria for the exclusion of 64 articles are described under the heading "Selection of studies" in the "Materials and methods" section

excluded because they were not obviously related to the purpose of this study (e.g., review articles, in vitro studies, and articles on drug delivery). Of the remaining 128 articles (plus 5 articles that were added after evaluation of citations within the 128 articles), 61 articles met the criteria for study selection. Table 1 shows summaries of these articles.

After 2000, many reports examined the possibilities of particle translocation to the systemic circulation, remote organs, or brain. Most reports before 2000 evaluated translocation to the lung interstitium or the lung-associated lymph nodes. As for particle materials, 29 kinds of materials were used in the articles analyzed in this report: 14 metals and their oxides and salts; 7 inorganic carbons and silicates; and 8 organic compounds. Polystyrene and its conjugates were the most frequently used (26 reports), at sizes that were usually $>100 \mathrm{~nm}$ in diameter. Titanium dioxide (18 reports) and inorganic carbons (16 reports) were also frequently used. In general, inorganic materials other than silica were frequent among particles whose diameters were $\leq 100 \mathrm{~nm}$, and plastics and silica were popular among particles of $>100 \mathrm{~nm}$. The smallest particle was C60 fullerene $(0.68 \mathrm{~nm})$, and the largest was polystyrene $(40 \mu \mathrm{m})$. As for the animal species, rat was the most frequently used (68 reports, approximately 60\%), followed by mouse (24 reports), dog (12 reports), and human (4 reports). All of the human reports were on inhalation of Technegas, and reported that no translocation to the systemic circulation or remote organs could be detected. As for the sites where particles were detected, there were 9 reports for the brain, 7 reports for the kidney, 3 reports for the heart, 2 reports for the thyroid, 20 reports for the liver, 4 reports for the spleen, 14 reports for blood, and 4 reports for lung capillary lumens. 
Table 1 Summaries of the selected studies on particle translocation

\begin{tabular}{|c|c|c|c|c|c|c|}
\hline Material & $\begin{array}{l}\text { Diameter } \\
(\mathrm{nm})\end{array}$ & Animal & Route & $\begin{array}{l}\text { Dose or } \\
\text { concentration }\end{array}$ & Results & Reference \\
\hline $\mathrm{Fe}_{3} \mathrm{O}_{4}$ & 1,300 & Rat & Inhalation & $\begin{array}{l}0.01,0.02,0.05 \\
0.1 \mathrm{mg} / \mathrm{m}^{3} \\
4 \text { weeks }\end{array}$ & Detected in lung-associated lymph node $[\mathrm{B}, \mathrm{C}]$ & [13] \\
\hline $\begin{array}{l}\text { Human serum } \\
\text { albumin }\end{array}$ & 7 & Rat & Intratracheal & $10 \mathrm{pmol} / \mathrm{g} \mathrm{wt}$ & $\begin{array}{l}\text { Detected in kidney, liver }[\mathrm{A}] \\
\text { and lymph node }[\mathrm{A}, \mathrm{D}]\end{array}$ & {$[14]$} \\
\hline mPEG $20 \mathrm{kDa}$ & 9 & Rat & Intratracheal & $10 \mathrm{pmol} / \mathrm{g} \mathrm{wt}$ & Detected in lymph node [D] & {$[14]$} \\
\hline $\begin{array}{l}\text { Polystyrene- } \\
\text { polyacrylate }\end{array}$ & 34 & Rat & Intratracheal & $10 \mathrm{pmol} / \mathrm{g} \mathrm{wt}$ & Detected in lymph node [D] & {$[14]$} \\
\hline $\begin{array}{l}\text { Polystyrene- } \\
\text { polyacrylate }\end{array}$ & 48 & Rat & Intratracheal & $10 \mathrm{pmol} / \mathrm{g} \mathrm{wt}$ & $<0.02 \%$ in lymph node & {$[14]$} \\
\hline $\begin{array}{l}\text { Polystyrene- } \\
\text { polyacrylate }\end{array}$ & 68 & Rat & Intratracheal & $10 \mathrm{pmol} / \mathrm{g} \mathrm{wt}$ & $<0.02 \%$ in lymph node & {$[14]$} \\
\hline $\begin{array}{l}\text { Polystyrene- } \\
\text { polyacrylate }\end{array}$ & 120 & Rat & Intratracheal & $10 \mathrm{pmol} / \mathrm{g} \mathrm{wt}$ & $<0.02 \%$ in lymph node & {$[14]$} \\
\hline $\begin{array}{l}\text { Polystyrene- } \\
\text { polyacrylate }\end{array}$ & 270 & Rat & Intratracheal & $10 \mathrm{pmol} / \mathrm{g} \mathrm{wt}$ & $<0.02 \%$ in lymph node & {$[14]$} \\
\hline $\mathrm{TiO}_{2}$ & 3 & Mouse & Intratracheal & $0.1 \mathrm{mg} \times 4$ & Detected in brain [B] & {$[15]$} \\
\hline $\mathrm{CeO}_{2}$ & 7 & Rat & Intratracheal & $0.2 \mathrm{mg}$ & $\begin{array}{l}\text { Detected in blood }[\mathrm{B}] .<0.01 \% \text { in heart, kidney, } \\
\text { brain, testis }\end{array}$ & {$[16]$} \\
\hline $\mathrm{Cu}$ & 24 & Mouse & Intranasal & $1 \mathrm{mg}, 40 \mathrm{mg}$ & $\begin{array}{l}\text { Detected in blood, liver, kidney, olfactory bulb } \\
\text { [B]. Not significant in spleen, heart, brain }\end{array}$ & {$[17]$} \\
\hline $\mathrm{Cu}$ & 17,000 & Mouse & Intranasal & $1 \mathrm{mg}$ & $\begin{array}{l}\text { Not significant in blood, liver, kidney, olfactory } \\
\text { bulb, spleen, heart, brain }\end{array}$ & {$[17]$} \\
\hline Polyacrylamide & 31 & Mouse & Intratracheal & $0.1 \mathrm{mg}$ & Detected in blood, liver, heart, thyroid [D] & {$[18]$} \\
\hline Polyacrylamide & 1,800 & Mouse & Intratracheal & $0.1 \mathrm{mg}$ & $\begin{array}{l}\text { Detected in blood, liver, spleen, kidney, heart, } \\
\text { thyroid [D] }\end{array}$ & {$[18]$} \\
\hline $\mathrm{Ir}$ & 20 & Rat & Inhalation & & $\begin{array}{l}\text { Detected in blood, liver, spleen, kidney, heart, } \\
\text { brain [D] }\end{array}$ & [19] \\
\hline Ir & 80 & Rat & Inhalation & & $\begin{array}{l}\text { Not detected in blood, liver, spleen, kidney, heart, } \\
\text { brain }\end{array}$ & [19] \\
\hline $\mathrm{TiO}_{2}$ & $700-800$ & Rat & Inhalation & $\begin{array}{r}2,10,50 \mathrm{mg} / \mathrm{m}^{3}, \\
6 \mathrm{~h} / \text { day, } 5 \text { days }\end{array}$ & $\begin{array}{l}\text { Detected in mediastinal lymph node [D]. Not } \\
\text { detected in liver, kidney, spleen, olfactory bulb, } \\
\text { brain }\end{array}$ & {$[20]$} \\
\hline Polystyrene & 100 & Mouse & Intranasal & $\begin{array}{r}6.8 \times 10^{8} \\
\text { particles }\end{array}$ & Detected in apical cells of olfactory epithelium [A] & {$[21]$} \\
\hline $\mathrm{C} 60$ & 0.68 & Mouse & Intratracheal & $0.625,1 \mathrm{mg}$ & $\begin{array}{l}\text { Detected in lung capillary, lymph nodes, type I } \\
\text { epithelial cells [A] }\end{array}$ & {$[22]$} \\
\hline $\mathrm{Au}$ & 2 & Mouse & Intratracheal & $3 \mathrm{mg}$ & Detected in liver [D] & {$[23]$} \\
\hline $\mathrm{Au}$ & 40 & Mouse & Intratracheal & $15 \mathrm{mg}$ & Not detected in liver & {$[23]$} \\
\hline $\mathrm{Au}$ & 100 & Mouse & Intratracheal & $15 \mathrm{mg}$ & Not detected in liver & {$[23]$} \\
\hline $\mathrm{TiO}_{2}$ & 1,100 & Rat & Inhalation & $\begin{array}{l}250 \mathrm{mg} / \mathrm{m}^{3}, 6 \mathrm{~h} / \\
\text { day, } 5 \text { days }\end{array}$ & Detected in mediastinal lymph node [D] & {$[24]$} \\
\hline Silica & 1,200 & Rat & Inhalation & $100 \mathrm{mg} / \mathrm{m}^{3}, 6 \mathrm{~h}$ & Detected in mediastinal lymph node [D] & {$[24]$} \\
\hline $\mathrm{TiO}_{2}$ & 80 & Mouse & Intranasal & $0.5 \mathrm{mg}$ & $\begin{array}{l}\text { Detected in olfactory bulb, hippocampus, cerebral } \\
\text { cortex, cerebellum [B] }\end{array}$ & {$[25]$} \\
\hline $\mathrm{TiO}_{2}$ & 150 & Mouse & Intranasal & $0.5 \mathrm{mg}$ & $\begin{array}{l}\text { Detected in olfactory bulb, hippocampus, cerebral } \\
\text { cortex, cerebellum [B] }\end{array}$ & {$[25]$} \\
\hline $\mathrm{TiO}_{2}$ & 21 & Rat & Intratracheal & $0.52 \mathrm{mg}$ & Detected in lymph node [B] & {$[26]$} \\
\hline $\mathrm{TiO}_{2}$ & 1,000 & Rat & Intratracheal & $10.7 \mathrm{mg}$ & Not significant in lymph node & {$[26]$} \\
\hline Carbon black & 120 & Rat & Inhalation & $\begin{array}{c}16 \mathrm{mg} / \mathrm{m}^{3} \\
4 \text { weeks }\end{array}$ & $\begin{array}{l}\text { Detected in endothelial cells of blood capillary } \\
\text { [A]. Not detected in liver, spleen, aortic } \\
\text { endothelial cells }\end{array}$ & [27] \\
\hline
\end{tabular}


Table 1 continued

\begin{tabular}{|c|c|c|c|c|c|c|}
\hline Material & $\begin{array}{l}\text { Diameter } \\
(\mathrm{nm})\end{array}$ & Animal & Route & $\begin{array}{l}\text { Dose or } \\
\text { concentration }\end{array}$ & Results & Reference \\
\hline $\begin{array}{l}\text { Carbon } \\
\text { (Technegas) }\end{array}$ & 100 & Human & Inhalation & & Not detected in liver & {$[28]$} \\
\hline $\mathrm{MnO}_{2}$ & 30 & Rat & Inhalation & $0.5 \mathrm{mg} / \mathrm{m}^{3}$ & $\begin{array}{l}\text { Detected in olfactory bulb, striatum, frontal cortex, } \\
\text { cerebellum [B] }\end{array}$ & [29] \\
\hline Polystyrene & 56 & Rat & Intratracheal & $0.6 \mathrm{mg}$ & Detected in liver, blood [D] & {$[30]$} \\
\hline Polystyrene & 202 & Rat & Intratracheal & $0.6 \mathrm{mg}$ & Detected in liver, blood [D] & {$[30]$} \\
\hline $\begin{array}{l}\text { Carbon } \\
\text { (Technegas) }\end{array}$ & 108 & Human & Inhalation & & No obvious uptake by liver or spleen & {$[31]$} \\
\hline Carbon black & 14 & Mouse & Intratracheal & $1 \mathrm{mg}$ & Detected in interstitium, capillary lumen [A] & {$[32]$} \\
\hline $\mathrm{Au}$ & 16 & Rat & Inhalation & $0.088 \mathrm{mg} / \mathrm{m}^{3}, 6 \mathrm{~h}$ & Detected in blood [B], alveolar epithelial cells [A] & {$[33]$} \\
\hline $\begin{array}{l}\text { Carbon } \\
\text { (Technegas) }\end{array}$ & 35 & Human & Inhalation & $\begin{array}{l}4.6 \times 10^{5} \\
\text { particles } / \mathrm{cm}^{3} \\
6 \text { min }\end{array}$ & No significant translocation to systemic circulation & [34] \\
\hline $\mathrm{TiO}_{2}$ & 22 & Rat & Inhalation & $0.11 \mathrm{mg} / \mathrm{m}^{3}$ & $\begin{array}{l}\text { Detected in capillary lumen [D], interstitium [A, } \\
\text { D], alveolar epithelial cells [D] }\end{array}$ & {$[35]$} \\
\hline Carbon black & 14 & Mouse & Intratracheal & $\begin{array}{l}(25,125, \\
\quad 625 \mu \mathrm{g}) \times 4\end{array}$ & Detected in mediastinal lymph node $[\mathrm{A}, \mathrm{B}, \mathrm{C}]$ & [36] \\
\hline Carbon black & 95 & Mouse & Intratracheal & $\begin{array}{l}(25,125, \\
625 \mu \mathrm{g}) \times 4\end{array}$ & Detected in mediastinal lymph node $[\mathrm{A}, \mathrm{B}, \mathrm{C}]$ & [36] \\
\hline Carbon & 36 & Rat & Inhalation & $0.16 \mathrm{mg} / \mathrm{m}^{3}$ & $\begin{array}{l}\text { Detected in olfactory bulb, cerebrum, cerebellum } \\
\text { [B] }\end{array}$ & [37] \\
\hline $\mathrm{Ir}$ & $15-20$ & Rat & Inhalation & $0.2 \mathrm{mg} / \mathrm{m}^{3}$ & Detected in liver, spleen, kidney, brain [D] & {$[38]$} \\
\hline $\mathrm{CdO}$ & 40 & Rat & Inhalation & $\begin{array}{l}0.07,0.55 \mathrm{mg} / \mathrm{m}^{3} \\
\quad 6 \mathrm{~h}\end{array}$ & Detected in liver, kidney [B] & [39] \\
\hline Polylactic acid & 1,500 & Rat & Intranasal & & Detected in submucosal layer $[\mathrm{A}]$ & {$[40]$} \\
\hline Polystyrene & 240 & Rat & Intratracheal & $9.5 \mathrm{mg}$ & $\begin{array}{l}\text { Not observed in alveolar epithelial cells, } \\
\text { endothelial cells, capillary lumen }\end{array}$ & [41] \\
\hline $\begin{array}{l}\text { Carbon } \\
\text { (Technegas) }\end{array}$ & 33 & Human & Inhalation & & No accumulation was observed in vicinity of liver & [42] \\
\hline $\mathrm{MnO}_{2}$ & 1,300 & Rat & Inhalation & $3 \mathrm{mg} / \mathrm{m}^{3}, 15$ days & $\begin{array}{l}\text { Detected in cerebral cortex [B]. Not significant in } \\
\text { liver, olfactory bulb, cerebellum, brainstem, } \\
\text { diencephalon, basal ganglia }\end{array}$ & [43] \\
\hline $\mathrm{MnO}_{2}$ & 18,000 & Rat & Inhalation & $3 \mathrm{mg} / \mathrm{m}^{3}, 15$ days & $\begin{array}{l}\text { Not significant in liver, olfactory bulb, cerebral } \\
\text { cortex, cerebellum, brainstem, diencephalon, } \\
\text { basal ganglia }\end{array}$ & [43] \\
\hline $\mathrm{Ir}$ & 15 & Rat & Inhalation & $2.5 \mathrm{mg} / \mathrm{m}^{3}, 1 \mathrm{~h}$ & Detected in liver [D] & [44] \\
\hline $\mathrm{Ir}$ & 80 & Rat & Inhalation & $2.5 \mathrm{mg} / \mathrm{m}^{3}, 1 \mathrm{~h}$ & $<0.1 \%$ in liver & [44] \\
\hline Carbon & $20-29$ & Rat & Inhalation & $\begin{array}{l}0.08,0.18 \mathrm{mg} / \mathrm{m}^{3} \\
\quad 6 \mathrm{~h}\end{array}$ & Detected in liver [B] & [45] \\
\hline $\mathrm{Ag}$ & 15 & Rat & Inhalation & $0.113 \mathrm{mg} / \mathrm{m}^{3}, 6 \mathrm{~h}$ & Detected in liver, kidney [D] & [46] \\
\hline $\mathrm{Ag}$ & 15 & Rat & Intratracheal & $0.05 \mathrm{mg}$ & Detected in liver [D] & [46] \\
\hline Polystyrene & 20 & Rat & Intranasal & $0.2 \mathrm{mg}$ & $\begin{array}{l}\text { Detected in blood [D]. }<0.1 \% \text { in liver, kidney, } \\
\text { spleen, brain }\end{array}$ & [47] \\
\hline Polystyrene & 100 & Rat & Intranasal & $0.2 \mathrm{mg}$ & $\begin{array}{l}\text { Detected in blood, liver [D]. }<0.1 \% \text { in kidney, } \\
\text { spleen, brain }\end{array}$ & [47] \\
\hline Polystyrene & 500 & Rat & Intranasal & $0.2 \mathrm{mg}$ & $\begin{array}{l}\text { Detected in blood, liver [D]. }<0.1 \% \text { in kidney, } \\
\text { spleen, brain }\end{array}$ & {$[47]$} \\
\hline Polystyrene & 1,000 & Rat & Intranasal & $0.2 \mathrm{mg}$ & $\begin{array}{l}\text { Detected in blood, liver [D]. }<0.1 \% \text { in kidney, } \\
\text { spleen, brain }\end{array}$ & [47] \\
\hline Polystyrene & 1,100 & Mouse & Intranasal & $1 \mathrm{mg}$ & $\begin{array}{l}\text { Detected in nasal-associated lymphoid tissues, } \\
\text { posterior lymph nodes, mediastinal lymph nodes } \\
{[\mathrm{A}, \mathrm{D}] .<0.1 \% \text { of lung burden in spleen }}\end{array}$ & {$[48]$} \\
\hline
\end{tabular}


Table 1 continued

\begin{tabular}{|c|c|c|c|c|c|c|}
\hline Material & $\begin{array}{l}\text { Diameter } \\
(\mathrm{nm})\end{array}$ & Animal & Route & $\begin{array}{l}\text { Dose or } \\
\text { concentration }\end{array}$ & Results & Reference \\
\hline $\begin{array}{l}\text { Colloidal } \\
\text { albumin }\end{array}$ & $<80$ & Hamster & Intratracheal & $0.1 \mathrm{mg}$ & $\begin{array}{l}\text { Detected in blood, liver }[\mathrm{D}] .<0.1 \% \text { in spleen, } \\
\text { kidney, brain }\end{array}$ & [49] \\
\hline Teflon & 18 & Rat & Inhalation & $\begin{array}{l}5 \times 10^{5} \text { particles/ } \\
\mathrm{cm}^{3}\end{array}$ & Detected in interstitium $[\mathrm{A}]$ & {$[50]$} \\
\hline Carbon & 25 & Mouse & Inhalation & & Detected in alveolar epithelial cells [A] & {$[50]$} \\
\hline $\mathrm{Pt}$ & 13 & Rat & Inhalation & $\begin{array}{l}5.6 \times 10^{6} \\
\text { particles } / \mathrm{cm}^{3}\end{array}$ & Detected in liver $[\mathrm{D}]$ & [50] \\
\hline $\mathrm{TiO}_{2}$ & 2,100 & Rat & Inhalation & $25,50 \mathrm{mg} / \mathrm{m}^{3}$ & Detected in lymph nodes [C] & {$[51]$} \\
\hline $\mathrm{BaSO}_{4}$ & 4,300 & Rat & Inhalation & $37.5,75 \mathrm{mg} / \mathrm{m}^{3}$ & Detected in lymph nodes [C] & {$[51]$} \\
\hline Polystyrene & 2,130 & Rat & Intratracheal & $\begin{array}{r}2.4 \times 10^{8} \\
\text { particles }\end{array}$ & Detected in lymph nodes [D] & {$[52]$} \\
\hline Mn phosphate & 4,900 & Rat & Intratracheal & $\begin{array}{r}40,80,160 \\
\mu \mathrm{g} \mathrm{Mn} / \mathrm{kg}\end{array}$ & No significant increase in striatum & {$[53]$} \\
\hline Mn phosphate & $1,670-15,500$ & Rat & Intratracheal & $\begin{array}{r}40,80,160 \\
\mu \mathrm{g} \mathrm{Mn} / \mathrm{kg}\end{array}$ & No significant increase in striatum & {$[53]$} \\
\hline $\mathrm{Mn}_{3} \mathrm{O}_{4}$ & 730 & Rat & Intratracheal & $\begin{array}{l}40,80, \\
160 \mu \mathrm{g} \mathrm{Mn} / \mathrm{kg}\end{array}$ & No significant increase in striatum & {$[53]$} \\
\hline Polystyrene & 40,000 & $\begin{array}{l}\text { Guinea } \\
\text { pig }\end{array}$ & Intratracheal & & Did not penetrate basement membrane & {$[54]$} \\
\hline Polystyrene & 830 & Rat & Intranasal & $8 \times 10^{9}$ particles & Detected in blood $[\mathrm{A}]$ & {$[55]$} \\
\hline $\mathrm{TiO}_{2}$ & 20 & Rat & Inhalation & $\begin{array}{l}23 \mathrm{mg} / \mathrm{m}^{3} \\
12 \text { weeks }\end{array}$ & Detected in lymph nodes, interstitium [D] & {$[56]$} \\
\hline $\mathrm{TiO}_{2}$ & 250 & Rat & Inhalation & $\begin{array}{r}23 \mathrm{mg} / \mathrm{m}^{3}, \\
12 \text { weeks }\end{array}$ & Detected in lymph nodes, interstitium [D] & {$[56]$} \\
\hline Silica & 300 & Mouse & Intratracheal & $1 \mathrm{mg}$ & Detected in interstitium $[\mathrm{A}, \mathrm{B}]$ & {$[57]$} \\
\hline $\mathrm{TiO}_{2}$ & 21 & Rat & Inhalation & $\begin{array}{r}23.5 \mathrm{mg} / \mathrm{m}^{3}, \\
12 \text { weeks }\end{array}$ & $\begin{array}{l}\text { Detected in hilar lymph nodes [D], alveolar } \\
\text { epithelial cells [A] }\end{array}$ & {$[58]$} \\
\hline $\mathrm{TiO}_{2}$ & 250 & Rat & Inhalation & $\begin{array}{l}23 \mathrm{mg} / \mathrm{m}^{3} \\
12 \text { weeks }\end{array}$ & $\begin{array}{l}\text { Detected in hilar lymph nodes [D], alveolar } \\
\text { epithelial cells [A] }\end{array}$ & {$[58]$} \\
\hline $\mathrm{TiO}_{2}$ & 20 & Rat & Intratracheal & $\begin{array}{l}0.065,0.1,0.2 \\
0.5,1 \mathrm{mg}\end{array}$ & Detected in epithelial cells/interstitium [C, D] & [59] \\
\hline $\mathrm{TiO}_{2}$ & 250 & Rat & Intratracheal & $0.5,1 \mathrm{mg}$ & Detected in epithelial cells/interstitium [D] & {$[59]$} \\
\hline $\mathrm{TiO}_{2}$ & 12 & Rat & Intratracheal & $0.5 \mathrm{mg}$ & Detected in epithelial cells/interstitium [D] & {$[59]$} \\
\hline $\mathrm{TiO}_{2}$ & 220 & Rat & Intratracheal & $0.5 \mathrm{mg}$ & Detected in epithelial cells/interstitium [D] & {$[59]$} \\
\hline Carbon black & 20 & Rat & Intratracheal & $0.5 \mathrm{mg}$ & Detected in epithelial cells/interstitium [D] & [59] \\
\hline Polystyrene & 1,700 & Rat & Intratracheal & $10^{7}, 10^{9}$ particles & Detected in interstitium $[\mathrm{A}, \mathrm{C}, \mathrm{D}]$ & {$[60]$} \\
\hline Polystyrene & 1,700 & Dog & Intratracheal & $10^{7}, 10^{9}$ particles & Detected in interstitium $[\mathrm{A}, \mathrm{D}]$ & {$[60]$} \\
\hline Carbon black & 240 & Rat & Inhalation & $\begin{array}{l}7 \mathrm{mg} / \mathrm{m}^{3}, 1,3 \\
6 \text { weeks }\end{array}$ & Detected in hilar lymph nodes $[C, D]$ & {$[61]$} \\
\hline Fly ash & 2,700 & Rat & Inhalation & $\begin{array}{l}1.7 \mathrm{mg} / \mathrm{m}^{3} \\
1 \text { year }\end{array}$ & $\begin{array}{l}\text { Particles less than } 2,300 \mathrm{~nm} \text { transported to } \\
\text { bronchopulmonary lymph nodes [A] }\end{array}$ & {$[62]$} \\
\hline Polystyrene & 1,900 & Rat & Intratracheal & $4 \times 10^{8}$ particles & Detected in tracheobronchial lymph nodes [A] & {$[63]$} \\
\hline Polystyrene & 1300 & Dog & Intratracheal & $\begin{array}{l}5 \times 10^{10} \\
\text { particles }\end{array}$ & Detected in lymph nodes $[\mathrm{A}, \mathrm{D}]$ & {$[64]$} \\
\hline $\mathrm{TiO}_{2}$ & 400 & Rat & Inhalation & $\begin{array}{l}10,50,250 \mathrm{mg} / \\
\mathrm{m}^{3}, 2 \text { years }\end{array}$ & $\begin{array}{l}\text { Detected in liver }[\mathrm{A}, \mathrm{C}] \text {, spleen }[\mathrm{A}, \mathrm{C}] \text {, lymph } \\
\text { node }[\mathrm{C}] \text {, type I epithelial cells }[\mathrm{A}]\end{array}$ & {$[65]$} \\
\hline Polystyrene & 3,000 & Dog & Intratracheal & $11 \mathrm{mg}$ & Detected in tracheobronchial lymph nodes [D] & {$[66]$} \\
\hline Polystyrene & 7,000 & Dog & Intratracheal & $0.7 \mathrm{mg}$ & Detected in tracheobronchial lymph nodes [D] & {$[66]$} \\
\hline Polystyrene & 13,000 & Dog & Intratracheal & $0.4 \mathrm{mg}$ & Not detected in tracheobronchial lymph nodes & {$[66]$} \\
\hline Aluminosilicate & 700 & Dog & Inhalation & IBB $11 \mu \mathrm{Ci}$ & Detected in lung-associated lymph nodes [D] & {$[67]$} \\
\hline Aluminosilicate & 1,500 & Dog & Inhalation & IBB $21 \mu \mathrm{Ci}$ & Detected in lung-associated lymph nodes [D] & [67] \\
\hline
\end{tabular}


Table 1 continued

\begin{tabular}{|c|c|c|c|c|c|c|}
\hline Material & $\begin{array}{l}\text { Diameter } \\
(\mathrm{nm})\end{array}$ & Animal & Route & $\begin{array}{l}\text { Dose or } \\
\text { concentration }\end{array}$ & Results & Reference \\
\hline Aluminosilicate & 2,800 & Dog & Inhalation & IBB $29 \mu \mathrm{Ci}$ & Detected in lung-associated lymph nodes [D] & {$[67]$} \\
\hline Aluminosilicate & 700 & Rat & Inhalation & IBB $0.2 \mu \mathrm{Ci}$ & $<0.1 \%$ in lung-associated lymph nodes & {$[67]$} \\
\hline Aluminosilicate & 1,500 & Rat & Inhalation & IBB $2.4 \mu \mathrm{Ci}$ & $<0.1 \%$ in lung-associated lymph nodes & {$[67]$} \\
\hline Aluminosilicate & 2,800 & Rat & Inhalation & IBB $0.43 \mu \mathrm{Ci}$ & $<0.1 \%$ in lung-associated lymph nodes & {$[67]$} \\
\hline Aluminosilicate & 700 & Mouse & Inhalation & IBB $0.075 \mu \mathrm{Ci}$ & $<0.1 \%$ in lung-associated lymph nodes & {$[67]$} \\
\hline Aluminosilicate & 1,500 & Mouse & Inhalation & IBB $0.56 \mu \mathrm{Ci}$ & $<0.1 \%$ in lung-associated lymph nodes & {$[67]$} \\
\hline Aluminosilicate & 2,800 & Mouse & Inhalation & IBB $2.5 \mu \mathrm{Ci}$ & $<0.1 \%$ in lung-associated lymph nodes & {$[67]$} \\
\hline Aluminosilicate & 1,700 & Dog & Intratracheal & ILB $0.5-64 \mu \mathrm{Ci}$ & Detected in thoracic lymph nodes [D] & {$[68]$} \\
\hline Silica & 1,400 & Rat & Inhalation & $109 \mathrm{mg} / \mathrm{m}^{3}$ & $\begin{array}{l}\text { Detected in alveolar epithelial cells, interstitium } \\
\text { [A] }\end{array}$ & [69] \\
\hline Carbon & 30 & Mouse & Intratracheal & $4 \mathrm{mg}$ & $\begin{array}{l}\text { Detected in type I alveolar epithelial cells and } \\
\text { interstitial cells [A] }\end{array}$ & {$[70]$} \\
\hline Polystyrene & 100 & Mouse & Intratracheal & $4 \mathrm{mg}$ & $\begin{array}{l}\text { Detected in interstitial cells and type I alveolar } \\
\text { epithelial cells [A] }\end{array}$ & {$[70]$} \\
\hline Polystyrene & 1,000 & Mouse & Intratracheal & $4 \mathrm{mg}$ & Detected in type I alveolar epithelial cells [A] & {$[70]$} \\
\hline $\begin{array}{l}\text { Colloidal } \\
\text { ferritin }\end{array}$ & 8 & Rat & Intratracheal & & Detected in vesicles in lymphatic endothelium [A] & [71] \\
\hline Carbon & 35 & Rat & Intratracheal & & $\begin{array}{l}\text { Detected in a vesicle within epithelial lining of } \\
\text { alveolar wall, vesicles within lymphatic } \\
\text { endothelium [A] }\end{array}$ & [71] \\
\hline $\mathrm{Au}$ & 30 & Rat & Intratracheal & $0.65 \mathrm{mg}$ & Detected in intracapillary platelets $[\mathrm{A}]$ & {$[72]$} \\
\hline${ }^{182} \mathrm{Ta}$ & 1,000 & Dog & Inhalation & ILB 30-7230 mg & Detected in lymph nodes $[\mathrm{A}, \mathrm{D}]$ & [73] \\
\hline${ }^{182} \mathrm{Ta}$ & 5,000 & Dog & Inhalation & $\begin{array}{l}\text { ILB } \\
2700-14000 \mathrm{mg}\end{array}$ & Detected in lymph nodes $[\mathrm{A}, \mathrm{D}]$ & {$[73]$} \\
\hline${ }^{182} \mathrm{Ta}$ & 10,000 & Dog & Inhalation & $\begin{array}{l}\text { ILB } \\
5240-5950 \mathrm{mg}\end{array}$ & Detected in lymph nodes [D] & [73] \\
\hline
\end{tabular}

[A]-[D] indicate the criteria for validity of particle detection (see " 5 . Validity of detection" under the heading "Selection of studies" in the "Materials and methods" section)

$w t$ weight, $I B B$ initial body burden, ILB initial lung burden, $m P E G$ methoxypoly (ethylene glycol)

\section{Statistical analysis of translocation}

\section{Evaluation of factors that affect translocation}

One characteristic of this study was that the factors associated with particle translocation from the airway were statistically evaluated using information contained in preceding systematic reviews. For this present study, CATREG analysis was conducted with 113 sets of available data found in 61 previous studies. The objective variable was the particle detection site, and the explanatory variables were particle diameter, particle material, animal species, and exposure route. The $F$ value of the model was $6.933(p<0.001)$. The coefficient of determination $\left(R^{2}\right)$ was 0.477 (unadjusted value; adjusted $R^{2}$ was 0.408 ), indicating that $<50 \%$ of the variance was explainable with these four variables. This model does not seem to be sufficient to predict particle translocation, but is reasonably acceptable to evaluate the relative importance of the explanatory variables for particle translocation. Table 2 summarizes the results of the analysis. The standardized partial regression coefficient $(\beta)$ values of all the explanatory variables were statistically significant. Both particle size and particle material showed large effects. On the other hand, the effects of exposure route and animal species were relatively small. Furthermore, Pratt's measure of relative importance, which is useful for comparing the contribution of each explanatory variable [74], also showed large contributions of particle size and particle material, followed by exposure route. The effect of animal species on particle translocation was very small.

\section{Effects of particle size}

As shown in Table 2, particle size was a strong factor for translocation. In addition, because particle size is a numerical variable, it can easily be expressed on the abscissa. Figure 2 shows the relationship between particle 
Table 2 Results of categorical regression analysis

\begin{tabular}{lllllr}
\hline $\begin{array}{l}\text { Explanatory } \\
\text { variable }\end{array}$ & $\begin{array}{l}\text { Scale (number } \\
\text { of categories) }\end{array}$ & \multicolumn{2}{l}{ Coefficient } & $p$ & \multirow{2}{*}{$\begin{array}{c}\text { Pratt's relative } \\
\text { importance }\end{array}$} \\
\cline { 3 - 4 } & Ordinal (5) & 0.427 & 0.131 & 0.000 & 0.43 \\
\hline Size & Nominal (6) & 0.441 & 0.102 & 0.000 & 0.49 \\
Material & Nominal (4) & 0.204 & 0.103 & 0.011 & -0.05 \\
Animal species & Nominal (3) & 0.258 & 0.093 & 0.001 & 0.14 \\
Route of exposure & & & & & \\
\hline
\end{tabular}
coefficient

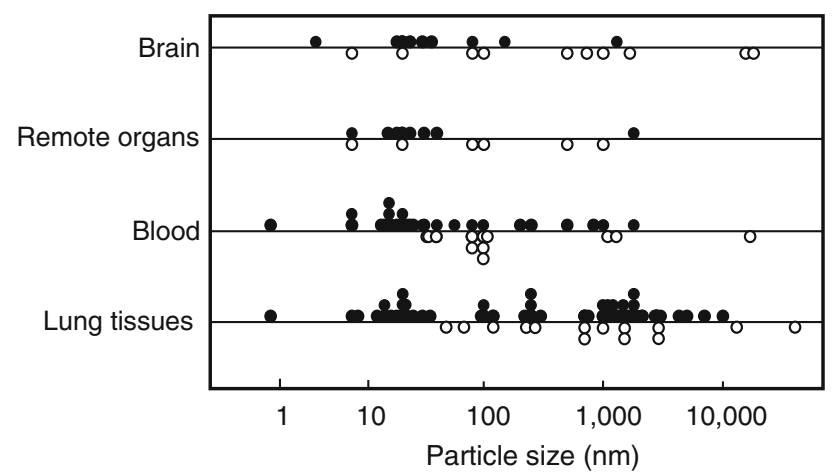

Fig. 2 Relationship between particle size and site of particle detection. Closed circles show the data of successful particle detection. Open circles show the data of negative results

size and detection site. Negative data for which particles were not detected are also included (shown by open circles in Fig. 2). Particles that were translocated to various sites were observed to have the following sizes: $\leq 50 \mathrm{~nm}$ for remote organs, $\leq 1 \mu \mathrm{m}$ for blood, and $\leq 10 \mu \mathrm{m}$ for lung tissues. In order to be detected in the blood, particles that have passed through the epithelial barrier must migrate into the capillaries. In addition, in order to be detected in remote organs, particles must be incorporated from the systemic blood circulation. Therefore, the conditions under which particles can be detected should be strictest for the remote organs, followed by the blood, and, finally, the lung tissues. In this context, it seems reasonable that detected particle size would be smallest for remote organs and largest for the lung tissues.

To further confirm the relationship between particle size and translocation, the odds ratios (ORs) for various cutoff points of size were calculated (Table 3). The OR was largest at the following cutoff points: $50 \mathrm{~nm}$ for brain and remote organs; $1 \mu \mathrm{m}$ for blood; and $10 \mu \mathrm{m}$ for lung tissues. The observations recorded in Fig. 2 were therefore also supported statistically.

\section{Translocation to the brain}

The largest $\mathrm{OR}$ for translocation to the brain was observed at a 50-nm cutoff size (Table 3). However, $\mathrm{MnO}_{2}$ particles as large as $1.3 \mu \mathrm{m}$ have also been
Table 3 Odds ratios for various cutoff points of particle diameter

\begin{tabular}{llllc}
\hline Cutoff point $(\mathrm{nm})$ & Brain & Remote organs & Blood & Lung tissues \\
\hline 50 & 8.0 & 9.3 & 6.3 & 5.4 \\
100 & 5.3 & 4.0 & 1.8 & 3.1 \\
1,000 & 3.4 & 0.4 & 9.0 & 0.9 \\
10,000 & 5.6 & 1.5 & 7.4 & 28.7 \\
\hline
\end{tabular}

detected in the cerebral cortex [43]. Translocation to the brain via the systemic circulation would be very difficult, as the blood-brain barrier (BBB) restricts intrusion into the brain. There are three possibilities as to why such large particles were detected in the brain. The first is that translocation to the brain occurs via a pathway other than the systemic circulation. The second is that Mn particles easily pass through the BBB. The third is that a small amount of soluble $\mathrm{Mn}$ was detected with a sensitive detection method.

As for the first possibility, Oberdörster et al. [9] advocated a mechanism in which particles deposited in the olfactory mucosa of the nasal cavity are subsequently taken into axons of the olfactory nerve and migrate into the olfactory bulb of the brain ventricle. In the present review, successful detection of particles in the brain was found mostly in data from inhalation and intranasal administration studies (8 sets of data), but in only one study of intratracheal administration [15]. The success rate of detection was 0.8 for inhalation and intranasal administration ( 8 successful detections out of 10 inhalations or administrations), but was less than 0.2 for intratracheal administration (1 successful detection in 6). This finding seems to support the hypothesis of Oberdörster et al., at least in part, that both inhalation and intranasal administration give particles a chance to contact olfactory nerves in the nasal cavity, while intratracheal administration, which skips the nasal cavity, does not. Although the statistical analysis suggested that the effect of the exposure route was relatively small for translocation to the brain, the exposure route might, nevertheless, be important.

As for the second and third possibilities, Yokel and Crossgrove [75] demonstrated that soluble Mn was transported into the brain via some transporting system(s) other 
than a divalent metal transporter (i.e., DMT-1). However, it is questionable as to whether $\mathrm{MnO}_{2}$ particles that exceed $1 \mu \mathrm{m}$ in diameter pass through the BBB with the help of some transporting system(s) similar to that for soluble Mn. On the other hand, various neurobehavioral effects have been reported among workers in the dry battery industry who had inhaled $\mathrm{MnO}_{2}$ aerosol [76, 77]; however, olfactory disorders were not specified among the workers. These findings suggest that the translocation of a substantial amount of $\mathrm{Mn}$ into the brain occurs via a route other than through the olfactory system. At present, the reason why 1.3- $\mu \mathrm{m} \mathrm{MnO}_{2}$ particles were detected in the brain remains unclear.

\section{Study limitations}

\section{Deficiency of information}

The statistical analysis conducted in the present study was based on currently available data; therefore, it is possible that the results of statistical evaluation will differ in the future when more information on particle translocation is accumulated. Particle materials and animal species varied, and some categories had only a few cases. Such types of information bias may have increased the uncertainty of the statistical analysis. Moreover, differences in crystal structures and fabrications of the particle surfaces were not considered because such information was limited.

\section{Publication bias}

This study was conducted based only on the information in published reports. There were 97 sets of data that reported successful detection of translocated particles. On the other hand, there were 42 sets of data indicating that particles were not detected. The degree of publication bias could not be evaluated objectively (e.g., by funnel plot) because of the characteristics of the collected data. Therefore, the possibility cannot be denied that publication bias was incorporated into the present review.

\section{Restriction by purpose of study}

There were several reports in which only neighboring tissues were examined because of the purposes of the studies, even though particles that could possibly translocate to distant tissues were used. It seemed, however, that a statistical analysis with plenty of data would make it possible to discern some broad trend, although some of the data in the reports mentioned above were included.

\section{Particle characteristics and detection}

Varying definitions of particle size and concentration were used (e.g., average and median for size, mass and particle number for concentration). In addition, size distribution can also vary among products. However, such differences were not considered. Particle detection was evaluated qualitatively; differences in the efficiency of translocation were not considered. Also, as for the method of particle detection, instead of morphological observation, the elements or labels of particles were measured in many studies. Regarding the measurement of elements or labels, the possibility cannot be ruled out that a translocated soluble fraction was detected. Reports with such a possibility were excluded by setting criteria on particle detection; however, it is possible that some papers that should have been excluded were included in this analysis.

\section{No consideration of interaction among the explanatory variables}

A lack of consideration of interaction among the explanatory variables may be one of the reasons why $R^{2}$ was less than 0.5 .

\section{No consideration of the effects caused by particles}

Based on the purpose of this study, any effects caused by the translocated particles were not considered.

\section{Conclusions}

This present study gives the results of a systematic review and statistical analysis of particle translocation from the respiratory system. A categorical regression analysis based on currently available data showed that all of the effects of particle size, particle material, animal species, and exposure route were statistically significant. The effects were large for particle size and particle material, and small for exposure route and animal species. These results suggest that, in an experiment to evaluate the translocation of solid particles, the characteristics of the particles (i.e., size and material) should be considered carefully. On the other hand, the selection of the types of experimental animals might be less important. When translocation to the brain is to be evaluated, inhalation and intranasal administration would be recommended. However, the results of this study should be considered within the context of several limitations. Similar analyses should be conducted when more information becomes available, especially related to the surface characteristics of particles. 
Acknowledgments The author thanks Dr. Hideo Tanaka of the Aichi Cancer Center Research Institute for helpful advice on statistical analyses.

Conflicts of interest The author belongs to the Atmospheric Environment Laboratory, Toyota Central R\&D Labs., Inc. This work has been conducted within the scope of the research plan of the company.

\section{References}

1. Brook RD, Franklin B, Cascio W, Hong Y, Howard G, Lipsett M, et al. A statement for healthcare professionals from expert panel on population and prevention science of the American Heart Association. Circulation. 2004;109:2655-71.

2. Peters A. Particulate matter and heart disease: evidence from epidemiological studies. Toxicol Appl Pharmacol. 2005;207: S477-82.

3. Pope CA III, Dockery DW. Health effects of fine particulate air pollution: lines that connect. J Air Waste Manag Assoc. 2006;56: 709-42.

4. Delfino RJ, Sioutas C, Malik S. Potential role of UFPs in associations between airborne particle mass and cardiovascular health. Environ Health Perspect. 2005;113:947-55.

5. Seaton A, MacNee W, Donaldson K, Godden D. Particulate air pollution and acute health effects. Lancet. 1995;345:176-8.

6. International Commission on Radiological Protection. Human respiratory tract model for radiological protection. (ICRP publication 66; Ann. ICRP 24). Oxford: Pergamon; 1994.

7. de Winter-Sorkina R, Cassee FR. From concentration to dose: factors influencing airborne particulate matter deposition in humans and rats. Report no. 650010031/2002. Retrieved September 29, 2009, from National Institute of Public Health and the Environment (RIVM) Website: http://www.rivm.nl/bibliotheek/ rapporten/650010031.html.

8. Gwinn MR, Vallyathan V. Nanoparticles: health effects-pro and cons. Environ Health Perspect. 2006;114:1818-25.

9. Oberdörster G, Oberdörster E, Oberdörster J. Nanotoxicology: an emerging discipline evolving from studies of ultrafine particles. Environ Health Perspect. 2005;113:823-39.

10. Elder A, Oberdörster G. Translocation and effects of ultrafine particles outside of the lung. Clin Occup Environ Med. 2006;5: 785-96.

11. Kreyling WG, Semmler-Behnke M, Möller W. Ultrafine particlelung interactions: does size matter? J Aerosol Med. 2006;19: 74-83.

12. Peters A, Veronesi B, Calderón-Garciudeñas L, Gehr P, Chen LC, Geiser M, et al. Translocation and potential neurological effects of fine and ultrafine particles a critical update. Particle Fiber Toxicol. 2006;3:13. doi:10.1186/1743-8977-3-13.

13. Pauluhn J. Subchronic inhalation toxicity of iron oxide (magnetite, $\mathrm{Fe}_{3} \mathrm{O}_{4}$ ) in rats: pulmonary toxicity is determined by the particle kinetics typical of poorly soluble particles. J Appl Toxicol. 2011. doi: 10.1002/jat.1668 (Article first published online: 1 APR 2011).

14. Choi HS, Ashitate Y, Lee JH, Kim SH, Matsui A, Insin N, et al. Rapid translocation of nanoparticles from the lung airspaces to the body. Nat Biotechnol. 2010;28:1300-3.

15. Li Y, Li J, Yin J, Li W, Kang C, Huang Q, et al. Systematic influence induced by $3 \mathrm{~nm}$ titanium dioxide following intratracheal instillation of mice. J Nanosci Nanotechnol. 2010;10: 8544-9.
16. He X, Zhang H, Ma Y, Bai W, Zhang Z, Lu K, et al. Lung deposition and extrapulmonary translocation of nano-ceria after intratracheal instillation. Nanotechnology. 2010;21. doi:10.1088/ 0957-4484/21/28/285103.

17. Liu Y, Gao Y, Zhang L, Wang T, Wang J, Jiao F, et al. Potential health impact on mice after nasal instillation of nano-sized copper particles and their translocation in mice. J Nanosci Nanotechnol. 2009;9:6335-43.

18. Liu Y, Ibricevic A, Cohen JA, Cohen JL, Gunsten SP, Fréchet $\mathrm{JM}$, et al. Impact of hydrogel nanoparticle size and functionalization on in vivo behavior for lung imaging and therapeutics. Mol Pharm. 2009;6:1891-902.

19. Kreyling WG, Semmler-Behnke M, Seitz J, Scymczak W, Wenk A, Mayer P, et al. Size dependence of the translocation of inhaled iridium and carbon nanoparticle aggregates from the lung of rats to the blood and secondary target organs. Inhal Toxicol. 2009;21(suppl.1):55-60.

20. Ma-Hock L, Burkhardt S, Strauss V, Gamer AO, Wiench K, van Ravenzwaay B, et al. Development of a short-term inhalation test in the rat using nano-titanium dioxide as a model substance. Inhal Toxicol. 2009;21:102-18.

21. Mistry A, Glud SZ, Kjems J, Randel J, Howard KA, Stolnik S, et al. Effect of physicochemical properties on intranasal nanoparticle transit into murine olfactory epithelium. J Drug Target. 2009; 17:543-52.

22. Naota M, Shimada A, Morita T, Inoue K, Takano H. Translocation pathway of the intratracheally instilled C60 fullerene from the lung into the blood circulation in the mouse: possible association of diffusion and caveolae-mediated pinocytosis. Toxicol Pathol. 2009;37:456-62.

23. Sadauskas E, Jacobsen NR, Danscher G, Stoltenberg M, Vogel U, Larsen A, et al. Biodistribution of gold nanoparticles in mouse lung following intratracheal instillation. Chem Cent J. 2009;3:16. doi:10.1186/1752-153X-3-16.

24. van Ravenzwaay B, Landsiedel R, Fabian E, Burkhardt S, Strauss $\mathrm{V}$, Ma-Hock L. Comparing fate and effects of three particles of different surface properties: nano- $\mathrm{TiO}_{2}$, pigmentary $\mathrm{TiO}_{2}$ and quartz. Toxicol Lett. 2009;186:152-9.

25. Wang J, Chen C, Liu Y, Jiao F, Li W, Lao F, et al. Potential neurological lesion after nasal instillation of $\mathrm{TiO}_{2}$ nanoparticles in the anatase and rutile crystal phases. Toxicol Lett. 2008;183:72-80.

26. Sager TM, Kommineni C, Castranova V. Pulmonary response to intratracheal instillation of ultrafine versus fine titanium dioxide: role of particle surface area. Particle Fiber Toxicol. 2008;5:17. doi:10.1186/1743-8977-5-17.

27. Niwa Y, Hiura Y, Sawamura H, Iwai N. Inhalation exposure to carbon black induces inflammatory response in rats. Circ J. 2008; 72:144-9.

28. Möller W, Felten K, Sommerer K, Scheuch G, Meyer G, Meyer $\mathrm{P}$, et al. Deposition, retention, and translocation of ultrafine particles from the central airways and lung periphery. Am J Respir Crit Care Med. 2008;177:426-32.

29. Elder A, Gelein R, Silva V, Feikert T, Opanashuk L, Carter J, et al. Translocation of inhaled ultrafine manganese oxide particles to the central nervous system. Environ Health Perspect. 2006;114: 1172-8.

30. Chen J, Tan M, Nemmar A, Song W, Dong M, Zhang G, et al. Quantification of extrapulmonary translocation of intratrachealinstilled particles in vivo in rats: effect of lipopolysaccharide. Toxicology. 2006;222:195-201.

31. Mills NL, Amin N, Robinson SD, Anand A, Davies J, Patel D, et al. Do inhaled carbon nanoparticles translocate directly into the circulation in humans? Am J Respir Crit Care Med. 2006; 173:426-31.

32. Shimada A, Kawamura N, Okajima N, Kaewamatawong T, Inoue $\mathrm{H}$, Morita T. Translocation pathway of the intratracheally 
instilled ultrafine particles from the lung into the blood circulation in the mouse. Toxicol Pathol. 2006;34:949-57.

33. Takenaka S, Karg E, Kreyling WG, Lentner B, Möller W, Behnke-Semmler M, et al. Distribution pattern of inhaled ultrafine gold particles in the rat lung. Inhal Toxicol. 2006;18:733-40.

34. Wiebert P, Sanchez-Crespo A, Falk R, Philipson K, Lundin A, Larsson $\mathrm{S}$, et al. No significant translocation of inhaled 35-nm carbon particles to the circulation in humans. Inhal Toxicol. 2006;18:741-7.

35. Geiser M, Rothen-Rutishauser B, Kapp N, Schürch S, Kreyling $\mathrm{W}$, Schulz $\mathrm{H}$, et al. Ultrafine particles cross cellular membranes by nonphagocytic mechanisms in lungs and in cultured cells. Environ Health Perspect. 2005;113:1555-60.

36. Shwe TT, Yamamoto S, Kakeyama M, Kobayashi T, Fujimaki H. Effect of intratracheal instillation of ultrafine carbon black on proinflammatory cytokine and chemokine release and mRNA expression in lung and lymph nodes of mice. Toxicol Appl Pharmacol. 2005;209:51-61.

37. Oberdörster G, Sharp Z, Atudorei V, Elder A, Gelein R, Kreyling $\mathrm{W}$, et al. Translocation of inhaled ultrafine particles to the brain. Inhal Toxicol. 2004;16:437-45.

38. Semmler M, Seitz J, Erbe F, Mayer P, Heyder J, Oberdörster G, et al. Long-term clearance kinetics of inhaled ultrafine insoluble iridium particles from the rat lung, including transient translocation into secondary organs. Inhal Toxicol. 2004;16:453-9.

39. Takenaka S, Karg E, Kreyling WG, Lentner B, Schulz H, Ziesenis A, et al. Fate and toxic effects of inhaled ultrafine cadmium oxide particles in the rat lung. Inhal Toxicol. 2004;16(suppl. 1):83-92.

40. Vila A, Sánchez A, Évora C, Soriano I, Vila Jato JL, Alonso MJ. PEG-PLA nanoparticles as carriers for nasal vaccine delivery. J Aerosol Med. 2004;17:174-85.

41. Kato T, Yashiro T, Murata Y, Herbert DC, Oshikawa K, Bando $\mathrm{M}$, et al. Evidence that exogenous substances can be phagocytized by alveolar epithelial cells and transported into blood capillaries. Cell Tissue Res. 2003;311:47-51.

42. Brown JS, Zeman KL, Bennett WD. Ultrafine particle deposition and clearance in the healthy and obstructed lung. Am J Respir Crit Care Med. 2002;166:1240-7.

43. Fechter LD, Johnson DL, Lynch RA. The relationship of particle size to olfactory nerve uptake of a non-soluble form of manganese into brain. Neurotoxicology. 2002;23:177-83.

44. Kreyling WG, Semmler M, Erbe F, Mayer P, Takenaka S, Schulz $\mathrm{H}$, et al. Translocation of ultrafine insoluble iridium particles from lung epithelium to extrapulmonary organs is size dependent but very low. J Toxicol Environ Health A. 2002;65:1513-30.

45. Oberdörster G, Sharp Z, Atudorei V, Elder A, Gelein R, Lunts A, et al. Extrapulmonary translocation of ultrafine carbon particles following whole-body inhalation exposure of rats. J Toxicol Environ Health A. 2002;65:1531-43.

46. Takenaka S, Karg E, Roth C, Schulz H, Ziesenis A, Heinzmann $\mathrm{U}$, et al. Pulmonary and systemic distribution of inhaled ultrafine silver particles in rats. Environ Health Perspect. 2001;109(suppl. 4):547-51.

47. Brooking J, Davis SS, Illum L. Transport of nanoparticles across the rat nasal mucosa. J Drug Target. 2001;9:267-79.

48. Eyles JE, Bramwell VW, Williamson ED, Alpar HO. Microsphere translocation and immunopotentiation in systemic tissues following intranasal administration. Vaccine. 2001;19:4732-42.

49. Nemmar A, Vanbilloen H, Hoylaerts MF, Hoet PHM, Berbruggen A, Nemery B. Passage of intratracheally instilled ultrafine particles form the lung into the systemic circulation in hamster. Am J Respir Crit Care Med. 2001;164:1665-8.

50. Oberdörster G, Finkelstein JN, Johnston C, Gelein R, Cox C, Baggs R, et al. Acute pulmonary effects of ultrafine particles in rats and mice. Res Rep Health Eff Inst. 2000;96:5-74.
51. Tran CL, Buchanan D, Cullen RT, Searl A, Jones AD, Donaldson $\mathrm{K}$. Inhalation of poorly soluble particles. II. Influence of particle surface area on inflammation and clearance. Inhal Toxicol. 2000;12:1113-26.

52. Trošić I, Mataušić-Pišl M, Horš N. Pathways and quantification of insoluble particles in the lung compartments of the rat. Int $\mathrm{J}$ Hyg Environ Health. 2000;203:39-43.

53. Vitarella D, Moss O, Dorman DC. Pulmonary clearance of manganese phosphate, manganese sulfate, and manganese tetraoxide by $\mathrm{CD}$ rats following intratracheal instillation. Inhal Toxicol. 2000;12:941-57.

54. Hunter DD, Undem BJ. Identification and substance P content of vagal afferent neurons innervating the epithelium of the guinea pig trachea. Am J Respir Crit Care Med. 1999;159:1943-8.

55. Alpar HO, Almeida AJ, Brown MRW. Microsphere absorption by the nasal mucosa of the rat. J Drug Target. 1994;2:147-9.

56. Oberdörster G, Ferin J, Lehnert BE. Correlation between particle size, in vivo particle persistence, and lung injury. Environ Health Perspect. 1994;102(suppl. 5):173-9.

57. Adamson IYR. Radiation enhances silica translocation to the pulmonary interstitium and increases fibrosis in mice. Environ Health Perspect. 1992;97:233-8.

58. Ferrin J, Oberdörster G, Penney DP. Pulmonary retention of ultrafine and fine particles in rats. Am J Respir Crit Care Med. 1992;6:535-42.

59. Oberdörster G, Ferin J, Gelein R, Soderholm SC, Finkelstein J. Role of the alveolar macrophage in lung injury: studies with ultrafine particles. Environ Health Perspect. 1982;97:193-9.

60. Mueller HL, Robinson B, Muggenburg BA, Gillett NA, Guilmette RA. Particle distribution in lung and lymph node tissues of rats and dogs and the migration of particle-containing alveolar cells in vitro. J Toxicol Environ Health. 1990;30:141-65.

61. Strom KA, Johnson JT, Chan TL. Retention and clearance of inhaled submicron carbon black particles. J Toxicol Environ Health. 1989;26:183-202.

62. Tanaka I. Particle size distributions in lungs and bronchopulmonary lymph nodes due to long-term exposure to coal fly ash aerosol in rats. J UOEH. 1987;9:361-7.

63. Lehnert BE, Valdez YE, Stewart CC. Translocation of particles to the tracheobronchial lymph nodes after lung deposition: kinetics and particle-cell relationships. Exp Lung Res. 1986;10:245-66.

64. Harmsen AG, Muggenburg BA, Snipes MB, Bice DE. The role of macrophages in particle translocation from lungs to lymph nodes. Science. 1985;230:1277-80.

65. Lee KP, Trochimowicz HJ, Reinhardt CF. Transmigration of titanium dioxide $\left(\mathrm{TiO}_{2}\right)$ particles in rats after inhalation exposure. Exp Mol Pathol. 1985;42:331-43.

66. Snipes MB, Chaves GT, Muggenburg BA. Disposition of 3-, 7-, and 13- $\mu \mathrm{m}$ microspheres instilled into lungs of dogs. Environ Res. 1984;33:333-42.

67. Snipes MB, Boecker BB, McClellan RO. Retention of monodisperse or polydisperse aluminosilicate particles inhaled by dogs, rats, and mice. Toxicol Appl Pharmacol. 1983;69:345-62.

68. Snipes MB, Muggenburg BA, Bice DE. Translocation of particles from lung lobes or the peritoneal cavity to regional lymph nodes in beagle dogs. J Toxicol Environ Health. 1983;11:703-12.

69. Brody AR, Roe MW, Evans JN, Davis GS. Deposition and translocation of inhaled silica in rats. Quantification of particle distribution, macrophage participation, and function. Lab Invest. 1982;6:533-42.

70. Adamson IYR, Bowden DH. Dose response of the pulmonary macrophagic system to various particulates and its relationship to transepithelial passage of free particles. Exp Lung Res. 1981;2:165-75.

71. Leak LV. Lymphatic removal of fluids and particles in the mammalian lung. Environ Health Perspect. 1980;35:55-76. 
72. Berry JP, Arnoux B, Stanislas G, Galle P, Chretien J. A microanalytic study of particles transport across the alveoli: role of blood platelets. Biomedicine. 1977;27:354-7.

73. Morrow PE, Kilpper RW, Beiter HE, Gibb FR. Pulmonary retention and translocation of insufflated tantalum. Radiology. 1976;121:415-21.

74. Pratt JW. Dividing the indivisible: using simple symmetry to partition variance explained. In: Pukkila T, Puntanen S, editors. Proceedings of the second international Tampere conference in statistics. Department of Mathematical Sciences, University of Tampere, Tampere; 1987. p. 245-260.
75. Yokel RA, Crossgrove JS. Manganese toxicokinetics at the blood-brain barrier. Res Rep Health Eff Inst. 2004;119:7-58.

76. Dietz MC, Ihrig A, Wrazidlo W, Bader M, Jansen O, Triebig G. Results of magnetic resonance imaging in long-term manganese dioxide-exposed workers. Environ Res. 2001;85:37-40.

77. Emara AM, El-Ghawabi SH, Madkour OI, El-Samra GH. Chronic manganese poisoning in the dry battery industry. Br J Ind Med. 1971;28:78-82. 